\title{
Cocycles of nilpotent quotients of free groups
}

\author{
Takefumi Nosaka1
}

\begin{abstract}
We focus on the cohomology of the $k$-th nilpotent quotient of the free group, $F / F_{k}$. This paper describes all the group 2-, 3-cocycles in terms of Massey product, and gives expressions for some of the 3-cocycles. We also give simple proofs of some of the results on Milnor invariant and the Johnson-Morita homomorphisms.
\end{abstract}

Keywords: nilpotent group, higher Massey product, group cohomology, mapping class group, link

\section{Contents}

1 Introduction

2 Review: Magnus expansions and higher Massey products

3 Main theorem: generators of $H^{*}\left(F / F_{k}\right)$

4 Applications: simple proofs

4.1 Theorem of Fenn-Sjerve on the Massey product . . . . . . . . . . . . . . 6

4.2 Milnor invariant and Massey product . . . . . . . . . . . . . . . . . 7

4.3 Johnson homomorphisms and Massey products . . . . . . . . . . . . 8

4.4 Vanishing condition of the Morita homomorphism . . . . . . . . . . . . 9 9

5 Expressions of some 3-cocycles 10

5.13 -cocycles of $F / F_{k} \ldots \ldots \ldots \ldots \ldots \ldots$

5.2 Quotient groups by central elements .................. . 11

A Cocycles in de Rham complexes of the iterated torus bundle 12

\section{Introduction}

Let $F$ be the free group of rank $q$. We define $F_{1}$ to be $F$, and $F_{k}$ to be the commutator subgroup $\left[F_{k-1}, F\right]$ by induction. Accordingly, we have the central extension,

$$
0 \longrightarrow F_{k} / F_{k+1} \longrightarrow F / F_{k+1} \stackrel{p_{k}}{\longrightarrow} F / F_{k} \longrightarrow 1 \quad \text { (central extension) }
$$

The abelian kernel $F_{k} / F_{k+1}$ is classically known to be free and of finite rank (see, e.g., [BC, MKS and references therein). We denote the rank by $N_{k} \in \mathbb{N}$.

The nilpotent quotient $F / F_{k}$ have been studied with applications; see, e.g., [Mas, BC, MKS] for the relation to the free Lie algebra $\oplus_{i=1}^{k} F_{k} / F_{k+1}$. The group homology of $F / F_{k}$ also plays a fruitful role in the study of low dimensional topology, including the Milnor (link) invariant, the Johnson-Morita homomorphisms, and tree parts of the quantum invariant; see GL, Ki, IO, Heap, KN, Mas, Tu, Po. Massey products of manifolds appear in nilpotent

\footnotetext{
${ }^{1}$ E-mail address: nosaka@math.titech.ac.jp
} 
obstructions of manifolds, as described in [CGO, FS, Ki, GL, Heap. Moreover, the homology groups of degree 2 and 3 are computed as

$$
H_{2}\left(F / F_{k} ; \mathbb{Z}\right) \cong \mathbb{Z}^{N_{k}}, \quad H_{3}\left(F / F_{k} ; \mathbb{Z}\right) \cong \bigoplus_{i=k}^{2 k-2} \mathbb{Z}^{q N_{i}-N_{i+1}}
$$

The former is a classical result; however, the latter was a result of Igusa and Orr [IO, Corollaries 5.5 and 6.5] shown by spectral sequences. In particular, it seems hard to deal with the third homology $H_{3}\left(F / F_{k} ; \mathbb{Z}\right)$ quantitatively.

In this paper, we describe bases of the cohomology $H^{2}\left(F / F_{k} ; \mathbb{Z}\right)$ and $H^{3}\left(F / F_{k} ; \mathbb{Z}\right)$ as Massey

products (see Theorem 3.2) and explicitly express some of the cocycles. Furthermore, we consider the Massey products to be an algorithm to produce many expressions of cocycles. In fact, Section 5 gives 3-cocycles of some nilpotent groups, and concretely describes their expressions. In doing so, it is reasonable to hope that the expressions may be useful for computing various things appearing in topology; including the Morita homomorphisms [M2] and the Orr link invariants [01]. Incidentally, the geometric realization of (1) is the iterated torus bundle; thus, in Appendix A, we can express the 2-cocycles as differential 2-forms in the de Rham complex; see Theorem A.4.

As corollaries, Section 4 provides simple proofs of four known results of [FS, Po, Tu, Heap, Ki , which are related to Massey products. The original proofs were discussed at the cohomology level, and they constructed Massey products according to circumstances. In contrast, since the above theorem ensures cocycles which gives a basis of $H^{*}\left(F / F_{k} ; \mathbb{Z}\right)$ as Massey products, we will give shorter proofs in terms of cocycles.

This paper is organized as follows. Section 2 reviews Magnus expansions and Massey products, and Section 3 states our theorem with a proof. Section 4 explains the four known results mentioned above and alternative proofs based on our theorem in Section 3. Section 5 discusses an algorithm to produce cocycles.

Conventional notation. Throughout this paper, let $F$ be the free group of rank $q$, and let $N_{k} \in \mathbb{Z}$ be the rank of $F_{k} / F_{k+1}$. Given a group $G$, we denote $G$ by $G_{1}$ and define $G_{k}$ to be the commutator subgroup $\left[G_{k-1}, G\right]$ by induction. For a group $G$, we write $B G$ for the Eilenberg-MacLane space, i.e., $K(G, 1)$-space. Furthermore, we assume the basic properties of group (co)-homology as in [Bro, Sections I, II, and VII].

\section{Review: Magnus expansions and higher Massey products}

Let us begin by studying unipotent Magnus expansions and higher Massey products.

First, we review the Magnus expansion modulo degree $k$. Let $\mathbb{Z}\left\langle X_{1}, \ldots, X_{q}\right\rangle$ be the polynomial ring with non-commutative indeterminates $X_{1}, \ldots, X_{q}$, and $\mathcal{J}_{k}$ be the two-sided ideal generated by polynomials of degree $\geq k$. Then, the Magnus expansion (of the free group $F$ ) is the multiplicative map $\mathcal{M}: F \rightarrow \mathbb{Z}\left\langle X_{1}, \ldots, X_{q}\right\rangle / \mathcal{J}_{k}$ defined by

$$
\mathcal{M}\left(x_{i}\right)=1+X_{i}, \quad \mathcal{M}\left(x_{i}^{-1}\right)=1-X_{i}+X_{i}^{2}+\cdots+(-1)^{k-1} X_{i}^{k-1} .
$$

As is known, $\mathcal{M}\left(F_{k}\right)=0$. By passage to this $F_{k}$, this $\mathcal{M}$ further induces the injection

$$
\mathcal{M}: F / F_{k} \longrightarrow \mathbb{Z}\left\langle X_{1}, \ldots, X_{k}\right\rangle / \mathcal{J}_{k}
$$


Next, we review another description of the Magnus embedding [GG], which is a faithful linear representation of $F / F_{k}$. Let $\Omega_{k}$ be the polynomial ring $\mathbb{Z}\left[\lambda_{i}^{(j)}\right]$ over commuting indeterminates $\lambda_{i}^{(j)}$ with $i \in\{1,2, \ldots, k-1\}, j \in\{1, \ldots, q\}$. We define the group homomorphism

$$
\Upsilon_{k}: F \longrightarrow G L_{k}\left(\Omega_{k}\right)
$$

by setting

$$
\Upsilon_{k}\left(x_{j}\right)=\left(\begin{array}{ccccc}
1 & \lambda_{1}^{(j)} & 0 & \ldots & 0 \\
0 & 1 & \lambda_{2}^{(j)} & \ldots & 0 \\
\vdots & \vdots & \ddots & \ddots & \vdots \\
0 & 0 & \ldots & 1 & \lambda_{k-1}^{(j)} \\
0 & 0 & \ldots & 0 & 1
\end{array}\right) .
$$

As is known [GG], the image $\Upsilon_{k}\left(F_{k}\right)$ consists of the identity matrix, and the quotient map $F / F_{k} \rightarrow G L_{k}\left(\Omega_{k}\right)$ is injective. Thus, we have an isomorphism $F / F_{k} \cong \operatorname{Im}\left(\Upsilon_{k}\right)$. It is shown [KN, Appendix] that the correspondence $1+X_{j} \mapsto \Upsilon_{k}\left(x_{j}\right)$ gives the equivalence beteween the former $\mathcal{M}$ and $\Upsilon_{k}$. The longer the word of $x$ is, the harder the computation of $\mathcal{M}(x)$ is; however, that of $\Upsilon_{k}(x)$ is still simpler. Indeed, the map $\Upsilon_{k}$ is defined over the commutative ring $\Omega_{k}$, and is therefore compatible with computer programs.

Next, we review the higher Massey products, which were first defined by Kraines [Kra]. Here, we describe the products in the non-homogenous complex of a group $G$ with a trivial coefficient ring $A$. That is, as in [Bro, Chap. III.1], we define the group $C^{*}(G ; A)$ of cochains to be $\operatorname{Map}\left(G^{n}, A\right)$ and the coboundary map $\partial_{n}^{*}$ by setting

$$
\begin{aligned}
& \left(\partial_{n}^{*} f\right)\left(g_{1}, \ldots, g_{n}\right)=f\left(g_{2}, \ldots, g_{n}\right)+(-1)^{n} f\left(g_{1}, \ldots, g_{n-1}\right) \\
& \quad-f\left(g_{1} g_{2}, g_{3}, \ldots, g_{n}\right)+f\left(g_{1}, g_{2} g_{3}, g_{4} \ldots, g_{n}\right)+\cdots+(-1)^{n-1} f\left(g_{1}, \ldots, g_{n-1} g_{n}\right) .
\end{aligned}
$$

Furthermore, the cup product on $C^{n}(G ; A)$ can be described as a canonical product. More precisely, for $u \in C^{p}(G ; A)$ and $v \in C^{q}(G ; A)$, the product $u \smile v \in C^{p+q}(G ; A)$ is defined by

$$
(u \smile v)\left(g_{1}, \ldots, g_{p+q}\right):=(-1)^{p q} u\left(g_{1}, \ldots, g_{p}\right) \cdot v\left(g_{p+1}, \ldots, g_{p+q}\right) \in A .
$$

For any $1 \leq i \leq n$, take a cocycle $\gamma_{i} \in C^{p_{i}}(G ; A)$. Then, a defining system associated with $\left(\gamma_{1}, \ldots, \gamma_{n}\right)$ is a set of elements $\left(a_{s, t}\right)$ for $1 \leq s \leq t \leq n$ with $(s, t) \neq(1, n)$, satisfying

(i) $a_{s, t} \in C^{p_{s}+p_{s+1}+\cdots+p_{t}-t+s}(G ; A)$.

(ii) When $s=t$, the diagonal map $a_{s, s}$ is equal to $\gamma_{s}$ in $C^{p_{s}}(G ; A)$.

(iii) $\partial^{*}\left(a_{s, t}\right)=\sum_{r=s}^{t-1}(-1)^{p_{s}+p_{t+1}+\cdots+p_{t}-t+s} a_{s, r} \smile a_{r+1, t}$.

Given such a defining system, we can define a cocycle of the form,

$$
\sum_{r: 1 \leq r \leq n-1}(-1)^{p_{1}+p_{2}+\cdots+p_{r}-r+1} a_{1, r} \smile a_{r+1, n} \in C^{p_{1}+p_{2}+\cdots+p_{n}-n+2}(G ; A) .
$$

Following [Kra], the $n$-fold Massey product, $\left\langle\gamma_{1}, \gamma_{2}, \ldots, \gamma_{n}\right\rangle$, is defined to be the set of cohomology classes of cocycles associated with all possible defining systems. While there are many interpretations of the higher Massey product, this paper uses the Massey products as a method to yield cocycles from other cocycles of lower degree.

Remark 2.1. As is known [FS], if $p_{1}=p_{2}=\cdots=p_{n}=1$ and every $m$-fold Massey products with $m<n$ chosen from $\left\{\gamma_{1}, \ldots, \gamma_{n}\right\}$ is null-cohomologous, the $n$-fold Massey product $\left\langle\gamma_{1}, \gamma_{2}, \ldots, \gamma_{n}\right\rangle$ is a singleton in $H^{2}(G ; A)$. 


\section{Main theorem: generators of $H^{*}\left(F / F_{k}\right)$}

We will describe bases of some cohomology of $F / F_{k}$ in terms of Massey products (Theorem 3.2).

Before stating the theorem, we should concretely define some defining systems. Let $F$ be the free group with basis, $x_{1}, \ldots, x_{q}$, and $G$ be $F / F_{k}$. For $1 \leq t \leq q$, let $\alpha_{t}: F / F_{k} \rightarrow \mathbb{Z}$ be the homomorphism which sends $x_{s}$ to $\delta_{s, t}$. We regard $\alpha_{t}$ as a 1-cocycle of $F / F_{k}$. Then, given a $k$-tuple $I=\left(i_{1}, \ldots, i_{k}\right) \in\{1,2, \ldots, q\}^{k}$, we have 1 -cocycles $\alpha_{i_{1}}, \ldots, \alpha_{i_{k}}$. Furthermore, for $1 \leq s \leq t \leq k$, let us consider the evaluation of the coefficient of $X_{i_{s}} \cdots X_{i_{t}}$. That is, we set up the linear map,

$$
\beta_{i_{s} i_{s+1} \cdots i_{t}}: \mathbb{Z}\left\langle X_{1}, \ldots, X_{q}\right\rangle \longrightarrow \mathbb{Z} ; \quad \sum a_{j_{1} \ldots j_{a}} X_{j_{1}} \cdots X_{j_{a}} \longmapsto a_{i_{s} i_{s+1} \ldots, i_{t}} .
$$

Let us denote the composite $\beta_{i_{s} \cdots i_{t}} \circ \mathcal{M}$ by $c_{i_{s} \cdots i_{t}}$, which we will use many times.

Lemma 3.1. Consider the case $p_{1}=p_{2}=\cdots=p_{n}=1$ with $n=k$. Let $a_{s, t}: F \rightarrow \mathbb{Z}$ be the composite $c_{i_{s} \cdots i_{t}}$. Then, the set of $\left(a_{s, t}\right)$ is a defining system associated with $\left(\alpha_{i_{1}}, \ldots, \alpha_{i_{k}}\right)$. In particular, the resulting 2-cocycle is represented by

$$
F / F_{k} \times F / F_{k} \longrightarrow \mathbb{Z} ; \quad(x, y) \longmapsto \sum_{\ell: 1 \leq \ell \leq k-1} c_{i_{1} i_{2} \cdots i_{\ell}}(x) c_{i_{\ell+1} \cdots i_{k}}(y) .
$$

Proof. From the unipotent Magnus expansion, the right hand side in (iii) is equivalent to the product of upper triangular matrices. Thus, it is not so hard to check (iii) by direct computation. Thus, the formula of the Massey products (41) readily means (6) $)$.

Moreover, let us review standard sequences from [CFL]. Equip the set of all sequences $\bigcup_{s=1}^{\infty}\{1,2, \ldots, q\}^{s}$ with the lexicographical order. Then, a sequence $I=i_{1} i_{2} \cdots i_{k}$ is said to be standard, if $I<i_{s} i_{s+1} \cdots i_{k}$ for any $2 \leq s \leq k$. For example, $\{123\}$ and $\{1223\}$ are standard, but $\{213\}$ and $\{3142\}$ are not standard. Let $\mathfrak{U}_{k}$ be the set of standard sequences of length $k$. As is known (see, e.g., [MKS] and [CFL, Theorem 1.5]), the order of $\mathfrak{U}_{k}$ is equal to $N_{k}$. Furthermore, the following is known:

$$
N_{k}:=\operatorname{rank}\left(F_{k} / F_{k+1}\right)=\operatorname{rank}\left(H^{2}\left(F / F_{k} ; \mathbb{Z}\right)\right)=\left|\mathfrak{U}_{k}\right|=\frac{1}{k} \sum_{d: d \mid k} \mu\left(\frac{k}{d}\right) q^{d} \in \mathbb{N},
$$

where $\mu$ is the Möbius function (see, e.g., Witt] or [CFL, Theorem 1.5]), and the last equality is commonly called Witt's formula.

While $F_{k} / F_{k+1}$ is classically known to be spanned by Hall basis (see, e.g., [Hall, MKS]) we will give a basis of $H^{2}\left(F / F_{k} ; \mathbb{Z}\right)$ for applications to the homology. Precisely, the second and third cohomology of $F / F_{k}$ are generated by Massey products:

Theorem 3.2. (I) Every $j$-fold Massey product with $j<k$ is zero. In particular, for any standard index $i_{1} \cdots i_{k} \in \mathfrak{U}_{k}$, the $k$-fold one $\left\langle\alpha_{i_{1}}, \ldots, \alpha_{i_{k}}\right\rangle$ is uniquely defined in $H^{2}\left(F / F_{k} ; \mathbb{Z}\right)$ and is represented by a 2-cocycle in (6) .

(II) The second cohomology $H^{2}\left(F / F_{k} ; \mathbb{Z}\right) \cong \mathbb{Z}^{N_{k}}$ is spanned by the $k$-fold Massey products $\left\langle\alpha_{i_{1}}, \ldots, \alpha_{i_{k}}\right\rangle$ running over all the standard sequences $\left(i_{1} \cdots i_{k}\right) \in \mathfrak{U}_{k}$.

(III) For any $k \leq \ell \leq 2 k-2$, consider the projection $p_{\ell}: F / F_{\ell} \rightarrow F / F_{k}$. Then, there are homomorphisms $\mathfrak{s}_{\ell}: Z^{3}\left(F / F_{\ell} ; \mathbb{Z}\right) \rightarrow Z^{3}\left(F / F_{k} ; \mathbb{Z}\right)$ such that

$$
\alpha_{s} \smile\left\langle\alpha_{i_{1}}, \ldots, \alpha_{i_{\ell}}\right\rangle=p_{\ell}^{*} \circ \mathfrak{s}_{\ell}\left(\alpha_{s} \smile\left\langle\alpha_{i_{1}}, \ldots, \alpha_{i_{\ell}}\right\rangle\right) \in Z^{3}\left(F / F_{\ell} ; \mathbb{Z}\right),
$$


for any $\left(i_{1} \cdots i_{\ell}\right) \in \mathfrak{U}_{\ell}, 1 \leq s \leq q$, and that the following set of 3-cocycles is a basis of the third cohomology $H^{3}\left(F / F_{k} ; \mathbb{Z}\right) \cong \oplus_{\ell=k}^{2 k-2} \mathbb{Z}^{q N_{\ell}-N_{\ell+1}}$.

$$
\bigcup_{k \leq \ell \leq 2 k-2}\left\{\mathfrak{s}_{\ell}\left(\alpha_{s} \smile\left\langle\alpha_{i_{1}}, \ldots, \alpha_{i_{\ell}}\right\rangle\right) \mid\left(i_{1} \cdots i_{\ell}\right) \in \mathfrak{U}_{\ell}, \quad 1 \leq s \leq q, \quad\left(i_{1} \cdots i_{\ell} s\right) \notin \mathfrak{U}_{\ell+1}\right\} .
$$

Remark 3.3. The statements of (I) (II) are classically known; see [FS, O2, Tu].

Proof. (I) Recall the definitions of (ii) and of (5). Thus, every lower Massey product is nullcohomologous by $a_{s, t}$ for some $(s, t)$, by induction on $k$.

(II) Denote by $\mathcal{S}$ the sum of $c_{i_{1} \cdots i_{k}}$ on $F_{k} / F_{k+1}$, where $i_{1} \cdots i_{k} \in \mathfrak{U}_{k}$. Namely, $\mathcal{S}:=\oplus_{I \in \mathfrak{U}_{k}} c_{I}$ : $F_{k} / F_{k+1} \rightarrow \mathbb{Z}^{N_{k}}$. As is known [CFL, Theorems 3.5 and 3.9], the sum $\mathcal{S}$ is surjective; hence, it is bijective. Accordingly, the centrally extended group operation on $F / F_{k} \times F_{k} / F_{k+1}$ from the 2-cocycles $\oplus_{I \in \mathfrak{U}_{k}}\left\langle\alpha_{i_{1}}, \ldots, \alpha_{i_{k}}\right\rangle$ is, by definition, formulated as

$$
(g, \alpha) \cdot(h, \beta)=\left(g h, \mathcal{S}^{-1}\left(\bigoplus_{i_{1} \cdots i_{k} \in \mathfrak{U}_{k}} c_{i_{1} i_{2} \cdots i_{k}}(\alpha+\beta)+\sum_{\ell: 1 \leq \ell<k} c_{i_{1} i_{2} \cdots i_{\ell}}(g) c_{i_{\ell+1} \cdots i_{k}}(h)\right)\right)
$$

for $g, h \in F / F_{k}$ and $\alpha, \beta \in F_{k} / F_{k+1}$. Here, it is worth noticing that the subsequences $i_{1} i_{2} \ldots i_{\ell}$, $i_{\ell+1} \cdots i_{k}$ are also standard. Therefore, via the Magnus expansion, this group is isomorphic to the nature $F / F_{k+1}$ as central extensions over $F / F_{k}$ (cf. matrix multiplications). Hence, the second cohomology $H^{2}\left(F / F_{k}\right) \cong \mathbb{Z}^{N_{k}}$ is generated by the sum $\oplus_{I \in \mathfrak{U}_{k}}\left\langle\alpha_{i_{1}}, \ldots, \alpha_{i_{k}}\right\rangle$, which provides a basis of $H^{2}\left(F / F_{k}\right)$, as desired.

(III) First, we will mention some of the results from [IO]. Igusa and Orr [IO, Theorem 6.7] constructed a certain filtration on the homology, $\mathcal{F}^{s} H_{3}\left(F / F_{k} ; \mathbb{Z}\right)$, such that two isomorphisms,

$$
\begin{aligned}
& \frac{\mathcal{F}^{\ell} H_{3}\left(F / F_{k} ; \mathbb{Z}\right)}{\mathcal{F}^{\ell+1} H_{3}\left(F / F_{k} ; \mathbb{Z}\right)} \cong \mathbb{Z}^{q N_{\ell-1}-N_{\ell}}, \\
& \mathcal{F}^{\ell} H_{3}\left(F / F_{k} ; \mathbb{Z}\right) \cong \operatorname{Im}\left(\left(p_{\ell-1}\right)_{*}: H_{3}\left(F / F_{\ell-1} ; \mathbb{Z}\right) \rightarrow H_{3}\left(F / F_{k} ; \mathbb{Z}\right)\right),
\end{aligned}
$$

hold for $k<\ell<2 k$, and the left quotients are zero otherwise. In particular, $\mathcal{F}^{\ell} H_{3}\left(F / F_{k} ; \mathbb{Z}\right)$ is a direct summand of $H_{3}\left(F / F_{k} ; \mathbb{Z}\right)$, and $\operatorname{Ker}\left(\left(p_{\ell-1}\right)_{*}\right)$ is a direct summand of $H_{3}\left(F / F_{\ell-1}\right)$, if $k<\ell<2 k$. Thus, for $\ell \geq k$, we readily have the composite,

$$
\left.H_{3}\left(F / F_{k} ; \mathbb{Z}\right)\right) \stackrel{\text { projection }}{\longrightarrow} \mathcal{F}^{\ell+1} H_{3}\left(F / F_{k}\right) \cong H_{3}\left(F / F_{\ell}\right) / \operatorname{Ker}\left(\left(p_{\ell}\right)_{*}\right) \hookrightarrow H_{3}\left(F / F_{\ell} ; \mathbb{Z}\right) .
$$

Applying of this composite to $\operatorname{Hom}(\bullet, \mathbb{Z})$ is regarded as a map $\iota_{\ell}: H^{3}\left(F / F_{\ell} ; \mathbb{Z}\right) \rightarrow H^{3}\left(F / F_{k} ; \mathbb{Z}\right)$. Accordingly, let us set up the composite of the cup product on $F / F_{\ell}$ and $\iota_{\ell}$ :

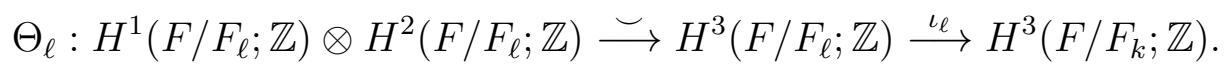

We will show that the direct sum $\oplus_{\ell=k}^{2 k-2} \Theta_{\ell}$ surjects onto $H^{3}\left(F / F_{k} ; \mathbb{Z}\right)$. Consider the LyndonHochschild spectral sequence from the central extension $F_{k} / F_{k+1} \rightarrow F / F_{k+1} \rightarrow F / F_{k}$. Then, as is shown in the proof of [IO, Lemma 5.8], we find a sequence,

$$
H_{3}\left(F / F_{k+1} ; \mathbb{Z}\right) \longrightarrow H_{3}\left(F / F_{k} ; \mathbb{Z}\right)=E_{3,0}^{2} \stackrel{d_{3,0}^{2}}{\longrightarrow} E_{1,1}^{2} \stackrel{\delta_{*}}{\longrightarrow} H_{2}\left(F / F_{k} ; \mathbb{Z}\right) \rightarrow 0 \quad \text { (exact), }
$$

satisfying $d_{3,0}^{2}\left(\mathcal{F}^{k+1} H_{3}\left(F / F_{k} ; \mathbb{Z}\right)\right)=\operatorname{Ker}\left(\delta_{*}\right)$ and $d_{3,0}^{2}\left(\mathcal{F}^{k} H_{3}\left(F / F_{k} ; \mathbb{Z}\right)\right)=0$. Keep in mind that each term is free. Furthermore, consider the spectral sequence on the cohomology level. Noting the identity

$$
E_{2}^{1,1}=H^{1}\left(F / F_{k} ; F_{k} / F_{k+1}\right) \cong H^{1}\left(F / F_{k} ; \mathbb{Z}\right) \otimes H^{2}\left(F / F_{k} ; \mathbb{Z}\right),
$$


we dually obtain the following sequence from (12):

$$
0 \longrightarrow H^{2}\left(F / F_{k}\right) \longrightarrow H^{1}\left(F / F_{k}\right) \otimes H^{2}\left(F / F_{k-1}\right) \stackrel{d_{2}^{3,0}}{\longrightarrow} \mathcal{F}^{k+1} H^{3}\left(F / F_{k}\right) \quad \text { (exact). }
$$

As is usual with the cup $_{1}$-product, the differential map $d_{2}^{3,0}$ is equal to the cup product. To summarize, this sequence and the isomorphisms (11) imply the surjectivity of $\oplus_{\ell=k}^{2 k-2} \Theta_{\ell}$, as required. Moreover, the construction of $\Theta_{\ell}$ admits the desired section $\mathfrak{s}_{\ell}$ satisfying (8).

The proof is completed by showing the linear independence of (9), as follows. Notice from the definition of $\left(i_{1} \cdots i_{\ell}\right) \in \mathfrak{U}_{\ell}$ that $\left(i_{1} \cdots i_{\ell} s\right) \in \mathfrak{U}_{\ell+1}$ if and only if $s>i_{1}$. Thus, if $s>i_{1}$, then $i_{\ell} i_{1} \cdots i_{\ell-1} s$ is not standard because of $i_{k} \geq s$. Here, we mention [GL, Proposition 4.5] showing the equality,

$$
\alpha_{s} \smile\left\langle\alpha_{i_{1}}, \ldots, \alpha_{i_{\ell}}\right\rangle=\alpha_{i_{\ell}} \smile\left\langle\alpha_{i_{1}}, \ldots, \alpha_{i_{\ell-1}}, \alpha_{s}\right\rangle \in H^{3}\left(F / F_{\ell} ; \mathbb{Z}\right) .
$$

Hence, $\alpha_{s} \smile\left\langle\alpha_{i_{1}}, \ldots, \alpha_{i_{\ell}}\right\rangle$ with $\left(i_{1} \cdots i_{\ell}\right) \in \mathfrak{U}_{\ell}$ and $\left(i_{1} \cdots i_{\ell} s\right) \in \mathfrak{U}_{\ell+1}$ is cohomologous to a cocycle in (9). By (8) and functoriality, a similar conclusion can be made even in the case $k<\ell \leq 2 k-2$. Hence, from the surjectivity of $\oplus_{\ell=k}^{2 k-2} \Theta_{\ell}$, comparing the ranks of $H^{3}\left(F / F_{k}\right)$ with the order of (9) leads to linear independence, as required.

We immediately obtain a corollary from the above proof.

Corollary 3.4. For any $s \leq q$, the map $H^{2}\left(F / F_{k} ; \mathbb{Z}\right) \rightarrow H^{3}\left(F / F_{k} ; \mathbb{Z}\right)$ which sends $\beta$ to $\alpha_{s} \smile \beta$ is injective.

Proof. The proof is straightforward from the basis in (9).

\section{Applications: simple proofs}

There are some topological invariants using $H^{*}\left(F / F_{k}\right)$, and some results on the Milnor linkinvariants and the mapping class groups of surfaces. In this section, we give simple proofs of the results of Fenn-Sjerve [FS], Turaev [Tu], Porter [Po], Kitano [Ki], and Heap [Heap].

\subsection{Theorem of Fenn-Sjerve on the Massey product}

We state the theorem [FS] and give an alternative proof using Theorem 3.2, Assume $k \geq 3$. Let $W_{1}, \ldots, W_{t}$ be words in $F_{k}$, and $R \subset F$ be the normal closure of $W_{1}, \ldots, W_{t}$ and $F_{k+1}$. Let $G$ be the quotient group $F / R$. Since $H_{1}(F) \cong H_{1}(G)$, the 1-cocycle $\alpha_{j}: F \rightarrow \mathbb{Z}$ induces the 1-cocycle $\alpha_{j}: G \rightarrow \mathbb{Z}$ for $j \leq q$.

We will state Theorem 4.1 below. Let $p: G \rightarrow F / F_{k}$ be the projection. Recall Hopf's theorem which claims the isomorphisms,

$$
H_{2}(G) \cong(R \cap[F, F]) /[F, R], \quad H_{2}\left(F / F_{k}\right) \cong\left(F_{k} \cap F_{2}\right) /\left[F, F_{k}\right]=F_{k} / F_{k+1} .
$$

Noticing $W_{j} \in R \cap[F, F]$, we denote the pushforward $p_{*}\left(W_{j}\right)$ by $\mathcal{W}_{j}$, and regard it as a 2-cycle of $F / F_{k}$.

Theorem 4.1 ([FS]). Suppose that all of $W_{1}, \ldots, W_{t}$ lie in $F_{k}$. For any $\ell<k$, every $\ell$-fold Massey product $\left\langle\alpha_{i_{1}}, \ldots, \alpha_{i_{\ell}}\right\rangle$ vanishes. On the other hand, Massey products of length $k$ are defined and evaluated on $\left\{W_{j}\right\}$ according to the formula,

$$
\sum_{j_{1}, \ldots, j_{k}}\left[\left\langle\alpha_{j_{1}}, \ldots, \alpha_{j_{k}}\right\rangle, W_{j}\right] X_{j_{1}} \cdots X_{j_{k}}=\mathcal{M}\left(\mathcal{W}_{j}\right) \in \mathcal{M}\left(F_{k} / F_{k+1}\right) .
$$

Here, the outer [,] is the pairing of $H^{2}(G ; \mathbb{Z})$ and $H_{2}(G ; \mathbb{Z})$. 
To prove this theorem, we give a lemma:

Lemma 4.2. Take a standard index $I=i_{1} \cdots i_{k} \in \mathfrak{U}_{k}$, and the associated Massey product $\left\langle\alpha_{i_{1}}, \ldots, \alpha_{i_{k}}\right\rangle$. Via the isomorphism $H_{2}\left(F / F_{k}\right) \cong F_{k} / F_{k+1}$ above, the Kronecker product $\left[\left\langle\alpha_{j_{1}}, \ldots, \alpha_{j_{k}}\right\rangle, \bullet\right]: H_{2}\left(F / F_{k}\right) \rightarrow \mathbb{Z}$ coincides with the map $c_{i_{1} \cdots i_{k}}: F_{k} / F_{k+1} \rightarrow \mathbb{Z}$.

Proof. For any $a \in F / F_{k}$, we choose a representative $\bar{a} \in F$. It follows from [Bro, Exercise 4 in II.5] that the correspondence $(g, h) \mapsto \bar{g} \bar{h}(\overline{g h})^{-1}$ induces the isomorphism $H_{2}\left(F / F_{k}\right) \rightarrow$ $F_{k} / F_{k+1}$. By the cocycle expression of $\left\langle\alpha_{j_{1}}, \ldots, \alpha_{j_{k}}\right\rangle$ in Lemma 3.1, a similar discussion to (10) readily deduces the required coincidence.

Proof of Theorem 4.1. Given a standard index $I=i_{1} \cdots i_{k} \in \mathfrak{U}_{k}$, let $W_{I} \in F_{k} / F_{k+1}$ be a generator of the $i_{1} \cdots i_{k}$-th summand of $\mathbb{Z}^{N_{k}} \cong F_{k} / F_{k+1}$. A previous paper [CFL, §2] explicitly describes the word $W_{I}$ and refers to it as the standard commutator. The previous paper CFL, Lemma 3.4] showed that, for any standard index $j_{1} \cdots j_{k}$, the $X_{j_{1}} \cdots X_{j_{k}}$-coefficient of $\mathcal{M}\left(W_{I}\right)$ is $\delta_{i_{1}, j_{1}} \cdots \delta_{i_{k}, j_{k}} \in\{0,1\}$. By Lemma 4.2, this is equal to $\left[\left\langle\alpha_{j_{1}}, \ldots, \alpha_{j_{k}}\right\rangle, \mathcal{W}_{I}\right]$. In summary, if $G$ is presented by $F /\left\langle F_{k+1}, W_{I}\right\rangle$, the equality (13) holds.

Finally, we complete the proof for $G=F / R$. Since $F_{k} / F_{k+1}$ is abelian, we can expand $p_{*}\left(W_{j}\right)$ as $\prod_{I \in \mathfrak{U}_{k}}\left(W_{I}\right)^{a_{I}}$ for some $a_{I} \in \mathbb{Z}$. Then, from the discussion in the above paragragh, the equality (13) holds in the $X_{j_{1}} \cdots X_{j_{k}}$-coefficient with respect to every standard index $j_{1} \cdots j_{k}$. Since any other coefficient is a linear sum of the such $X_{j_{1}} \cdots X_{j_{k}}$-coefficients, we conclude that the equality (13) in $\mathcal{M}\left(F_{k} / F_{k+1}\right)$ is satisfied.

Remark 4.3. Finally, we should give a remark for the following subsections. Let us choose a connected CW complex $X$ such that $\pi_{1}(X) \cong G$, and fix a classifying map $c: X \rightarrow B G$ which is obtained by killing the higher homotopy groups of $X$. Since $c_{*}: H_{2}(X) \rightarrow H_{2}(B G ; \mathbb{Z})=H_{2}(G)$ is surjective, there are 2-cycles $\mathcal{X}_{j} \in H_{2}(X)$ with $c_{*}\left(\mathcal{X}_{j}\right)=\left[W_{j}\right]$. By $\pi_{1}(X) \cong G$, the 1-cocycle $\alpha_{j}$ may be that of $H^{1}(X ; \mathbb{Z})$, and the pairing $\left[\left\langle\alpha_{j_{1}}, \ldots, \alpha_{j_{k}}\right\rangle, \mathcal{X}_{j}\right]$ is equal to the equality (13). To conclude, we can deal with some Massey products in $H^{2}(X ; \mathbb{Z})$ from the viewpoits of Theorem 4.1 .

\subsection{Milnor invariant and Massey product}

Porter [Po] and Turaev [Tu] independently showed that the Milnor link invariant is equivalent to some Massey products of the link complement space. For simplicity, this paper focuses on only the $k$-th leading terms of the Milnor invariant and gives an alternative proof of their result.

To state the theorems, we begin by reviewing the Milnor invariant, according to [Mil, Tu]. We suppose that the reader has elementary knowledge of knot theory, as provided in $[\mathrm{Po}, \mathrm{Tu}]$. Let $M$ be an integral homology 3 -sphere, that is, a closed 3 -manifold such that $H_{*}(M ; \mathbb{Z}) \cong$ $H_{*}\left(S^{3} ; \mathbb{Z}\right)$. Choose a link $L \subset M$ with $q$ components, and a meridian-longitude pair $\left(\mathfrak{m}_{\ell}, \mathfrak{l}_{\ell}\right)$ for $\ell \leq q$, where $\mathfrak{l}_{\ell}$ may be the preferred longitude. Then, it is known ([Mil]; see also [Tu, Lemma 1.2]) that the $m$-th nilpotent quotient $\pi_{1}(M \backslash L) / \pi_{1}(M \backslash L)_{m}$ has the group presentation,

$$
\left.\left\langle x_{1}, \ldots, x_{q}\right|\left[x_{\ell}, w_{\ell}^{(m)}\right]=1 \text { for } \ell \leq q, F_{m}\right\rangle
$$

where $x_{i}$ is represented by the $j$-th meridian, and $w_{j}^{(m)}$ is defined by the longitude in $\pi_{1}(M \backslash L)$.

For brevity, let us assume the existence of $k \in \mathbb{Z}$ such that $w_{j}^{(m)}$ is trivial in the $k$-th quotient for any $m \leq k$, i.e., $w_{j}^{(k)} \in F_{k}$. We call the existence Assumption $\mathcal{A}_{k}$. By considering 
$w_{\ell}^{(k)}$ to be a word in $F_{k} / F_{k+1}$, we will focus on the value $\mathcal{M}\left(w_{\ell}^{(k)}\right) \in \mathbb{Z}\left\langle X_{1}, \ldots, X_{q}\right\rangle / \mathcal{J}_{k+1}$. The coefficient of $X_{i_{1}} \cdots X_{i_{k}}$ of $\mathcal{M}\left(w_{\ell}^{(k)}\right)$ is called the $k$-th Milnor $\mu$-invariant of $L$, and it is denoted by $\mu\left(i_{1} \cdots i_{k} ; \ell\right)$. Let $\alpha_{j}: \pi_{1}(M \backslash L ; \mathbb{Z}) \rightarrow \mathbb{Z}$ be the homomorphism which sends $\mathfrak{m}_{\ell}$ to $\delta_{\ell, j}$.

Theorem 4.4 (The minimal non-vanishing case of $[\mathrm{Tu}][\mathrm{Po}])$. Suppose $\mathcal{A}_{k}$. Let $\left[\mathfrak{l}_{\ell}\right] \in H_{2}(M \backslash$ $L ; \mathbb{Z})$ be a 2-cycle corresponding to the $\ell$-th longitude $\mathfrak{l}_{\ell}$. For any index $I=i_{1} i_{2} \cdots i_{k}$, the $(k+$ 1)-fold Massey product $\left\langle\alpha_{i_{1}}, \ldots, \alpha_{i_{k-1}}, \alpha_{i_{k}}, \alpha_{\ell}\right\rangle$ is uniquely defined, and the following equality holds:

$$
\mu\left(i_{1} \cdots i_{k} ; \ell\right)=\left[\left\langle\alpha_{i_{1}}, \ldots, \alpha_{i_{k-1}}, \alpha_{i_{k}}, \alpha_{\ell}\right\rangle,\left[\mathfrak{l}_{\ell}\right]\right] \in \mathbb{Z}
$$

Proof. Denote $\pi_{1}(M \backslash L)$ by $G$, and take the classifying map $\mathcal{C}: M \backslash L \rightarrow B G$, explained in Remark 4.3. We claim that the pushforward $\mathcal{C}_{*}\left(\left[\mathfrak{l}_{\ell}\right]\right)$ corresponds to the relation $\left[\mathfrak{m}_{\ell}, \mathfrak{l}_{\ell}\right]$. Consider the $\ell$-th torus boundary, $\partial_{\ell}(M \backslash L)$, as a $K\left(\mathbb{Z}^{2}, 1\right)$-space. This $\pi_{1}\left(\partial_{\ell}(M \backslash L)\right) \cong \mathbb{Z}^{2}$ is presented by $\left\langle\mathfrak{m}_{\ell}, \mathfrak{l}_{\ell} \mid \mathfrak{m}_{\ell} \mathfrak{l}_{\ell} \mathfrak{m}_{\ell}^{-1} \mathfrak{l}_{\ell}^{-1}\right\rangle$. Following Hopf's theorem, the generator of $H_{2}\left(\partial_{\ell}(M \backslash L)\right) \cong$ $\mathbb{Z}$ corresponds to $\mathfrak{m}_{\ell} \mathfrak{l}_{\ell} \mathfrak{m}_{\ell}^{-1} \mathfrak{l}_{\ell}^{-1}$. Considering the pushforwards via the inclusion $\partial_{\ell}(M \backslash L) \rightarrow$ $M \backslash L$, we will prove the claim using $\mathcal{C}$. Let $p: G \rightarrow G / G_{k+1}$ be the projection. By the above claim, the pushforward $(p \circ \mathcal{C})_{*}\left(\left[\mathfrak{l}_{\ell}\right]\right)$ corresponds to the word $\left[x_{\ell}, w_{\ell}^{(k)}\right]$.

We are now in a position to complete the proof. By the assumption, $w_{\ell}^{(k)}$ lies in $F_{k}$. Therefore, $\left[x_{\ell}, w_{\ell}^{(k)}\right] \in F_{k+1}$. Hence, it follows from the theorem 4.1 of Fenn-Sjerve that $\left\langle\alpha_{i_{1}}, \ldots, \alpha_{i_{k}}, \alpha_{\ell}\right\rangle$ of length $k+1$ is uniquely defined; see Remark 2.1. Moreover, the left hand side of (15) equals the right hand side of (13), and the right one means the $X_{i_{1}} \cdots X_{i_{k}} X_{\ell^{-}}$ coefficient of $\mathcal{M}\left(\left[x_{\ell}, w_{\ell}^{(k+1)}\right]\right)$. Then, we can verify, by directly computing $\mathcal{M}\left(x_{\ell} w_{\ell}^{(k+1)} x_{\ell}^{-1}\left(w_{\ell}^{(k+1)}\right)^{-1}\right)$, that it is equal to the $X_{i_{1}} \cdots X_{i_{k}}$-coefficient of $\mathcal{M}\left(w_{\ell}^{(k)}\right)$, that is, $\mu\left(i_{1} \cdots i_{k} ; \ell\right)$, as required.

Incidentally, Milnor defined the $\mu$-invariant of higher degree, and Porter [Po] and Turaev [Tu] described the higher invariant in terms of Massey product; see also the paper [KN], which provides a refinement of the higher invariant. Although we omit showing the details, the higher degree relation can be proven in the same manner as Theorem 4.4 .

\subsection{Johnson homomorphisms and Massey products}

Now let us focus on the Johnson homomorphisms of the mapping class groups of surfaces; see, e.g., [Joh1, M1, Day, GL, Heap, M2] for the significance of these homomorphisms. This section paraphrases [Ki], which describes the relation between the Johnson homomorphisms and Massey products.

First, we should establish the notation. Suppose $q=2 g$. Let $\Sigma_{g, r}$ be a compact oriented surface of genus $g$ with $r$ boundaries. Let $\Gamma_{g, 1}$ be the group of isotopy classes of orientationpreserving homeomorphisms of $\Sigma_{g, 1}$. Then, the action of $\Gamma_{g, 1}$ on $F=\pi_{1}\left(\Sigma_{g, 1}\right)$ can be regarded as a homomorphism $\Gamma_{g, 1} \rightarrow \operatorname{Aut}(F)$. Subject to $F_{k}$, we have $\rho_{k}: \Gamma_{g, 1} \rightarrow \operatorname{Aut}\left(F / F_{k}\right)$. We commonly denote the kernel $\operatorname{Ker}\left(\rho_{k}\right)$ by $\mathcal{T}(k)$. We have the filtration $\Gamma_{g, 1} \supset \mathcal{T}(2) \supset \mathcal{T}(3) \supset$ $\cdots$.

Next, let us review the homomorphism (16) below. Fix any $f \in \mathcal{T}(k)$. Given $[\gamma] \in$ $H_{1}\left(\Sigma_{g, 1}\right)=F / F_{2}$, choose a representative $\gamma \in \pi_{1}\left(\Sigma_{g, 1}\right)$. Then, $f_{*}(\gamma) \gamma^{-1}$ lies in $F_{k}$ since the action of $f_{*}$ by $f \in \mathcal{T}(k)$ on $F / F_{k}$ is trivial. Then, the $k$-th Johnson homomorphism is defined as the map,

$$
\tau_{k}: \mathcal{T}(k) \longrightarrow \operatorname{Hom}\left(H_{1}\left(\Sigma_{g, 1}\right), \quad F_{k} / F_{k+1}\right)
$$


which sends $[f]$ to the homomorphism $[\gamma] \rightarrow\left[f_{*}(\gamma) \gamma^{-1}\right]$. As is known, this $\tau_{k}$ is well-defined and a homomorphism. The following is also well-known; see [Joh2, M2]. For $f \in \mathcal{T}(k)$, this $f$ lies in $\mathcal{T}(k+1)$ if and only if $\tau_{m}(f)=0$ for any $m \leq k$.

Next, let us examine the mapping torus $T_{f, 1}$ for a fixed $f \in \mathcal{T}(k)$ with $k \geq 2$. Here, $T_{f, 1}$ is the quotient space of $\Sigma_{g, 1} \times[0,1]$ subject to the relation $(y, 0) \sim(f(y), 1)$ for any $y \in \Sigma_{g, 1}$. Since $f \in \mathcal{T}(k)$ with $k \geq 2$, we have $H_{*}\left(T_{f, 1}\right) \cong H_{*}\left(\Sigma_{g, 1} \times S^{1}\right)$. Furthermore, fix a basis $\left\{x_{1}, \ldots, x_{2 g}\right\}$ of the free group $F=\pi_{1}\left(\Sigma_{g, 1}\right)$. Then, following the van Kampen argument, one can verify the presentation,

$$
\pi_{1}\left(T_{f, 1}\right) \cong\left\langle x_{1}, \ldots, x_{2 g}, \gamma \mid\left[x_{1}, \gamma\right] f_{*}\left(x_{1}\right) x_{1}^{-1}, \ldots,\left[x_{2 g}, \gamma\right] f_{*}\left(x_{2 g}\right) x_{2 g}^{-1}\right\rangle .
$$

Here, $\gamma$ represents a generator of $\pi_{1}\left(S^{1}\right)$. Since $T_{f, 1}$ is a $\Sigma_{g, 1}$-bundle over $S^{1}$ by definition, it is a $K(\pi, 1)$-space. Hence, $H_{*}\left(\pi_{1}\left(T_{f, 1}\right)\right) \cong H_{*}\left(T_{f, 1}\right) \cong H_{*}\left(\Sigma_{g, 1} \times S^{1}\right)$. Moreover, Hopf's theorem implies that the relations $\left[x_{1}, \gamma\right] f_{*}\left(x_{1}\right) x_{1}^{-1}, \ldots,\left[x_{2 g}, \gamma\right] f_{*}\left(x_{2 g}\right) x_{2 g}^{-1}$ represent a basis $\left\{\mathcal{X}_{1}, \ldots, \mathcal{X}_{2 g}\right\}$ of $H_{2}\left(T_{f, 1}\right) \cong \mathbb{Z}^{2 g}$.

Now let us state and prove Proposition 4.5. Since the boundary $\partial T_{f, 1}$ is the torus $\partial \Sigma_{g, 1} \times S^{1}$, we can define $T_{f}^{\gamma}$ to be the resulting space obtained by filling in the torus $\partial T_{f, 1} \simeq \partial \Sigma_{g, 1} \times S^{1}$ with the solid torus $\partial \Sigma_{g, 1} \times D^{2}$. This $T_{f}^{\gamma}$ is called the Dehn filling along a curve on $\partial T_{f, 1}$. By denoting the inclusion $T_{f, 1} \rightarrow T_{f}^{\gamma}$ by $\iota^{\gamma}$, the homology $H_{2}\left(T_{f}^{\gamma} ; \mathbb{Z}\right) \cong H^{1}\left(T_{f}^{\gamma} ; \mathbb{Z}\right) \cong \mathbb{Z}^{2 g}$ is spanned by the pushforwards $\left\{\iota_{*}^{\gamma}\left(\mathcal{X}_{1}\right), \ldots, \iota_{*}^{\gamma}\left(\mathcal{X}_{2 g}\right)\right\}$. Moreover, we should notice the presentation of $\pi_{1}\left(T_{f}^{\gamma}\right)$

$$
\pi_{1}\left(T_{f}^{\gamma}\right) \cong\left\langle x_{1}, \ldots, x_{2 g} \mid f_{*}\left(x_{1}\right) x_{1}^{-1}, \ldots, f_{*}\left(x_{2 g}\right) x_{2 g}^{-1}\right\rangle .
$$

Proposition 4.5 (cf. [Ki] and [GL, Corollary 4.1]). Let $\left\{\mathcal{X}_{1}, \ldots, \mathcal{X}_{2 g}\right\}$ be the basis of $H_{2}\left(T_{f, 1}\right)$, and $\iota^{\gamma}: T_{f, 1} \rightarrow T_{f}^{\gamma}$ be the inclusion as above. Let $x_{j}^{*}: \pi_{1}\left(T_{f}^{\gamma}\right) \rightarrow \mathbb{Z}$ be the 1-cocycle which sends $x_{i}$ to $\delta_{j, i}$. Define the map $\tau_{k}^{\prime}: \mathcal{T}(k) \rightarrow \operatorname{Hom}\left(H_{1}\left(\Sigma_{g, 1}\right), \mathcal{M}\left(F_{k} / F_{k+1}\right)\right)$ by letting $\tau_{k}^{\prime}(f)$ be the homomorphism,

$$
x_{i} \longmapsto \sum_{j_{1}, \ldots, j_{k}}\left[\left\langle x_{j_{1}}^{*}, \ldots, x_{j_{k}}^{*}\right\rangle, \iota_{*}^{\gamma}\left(\mathcal{X}_{i}\right)\right] X_{j_{1}} \cdots X_{j_{k}} .
$$

Here, the outer $[$,$] is the pairing of H^{2}\left(T_{f}^{\gamma}\right)$ and $H_{2}\left(T_{f}^{\gamma}\right)$. Then, $\tau_{k}^{\prime}(f)\left(x_{i}\right)$ is equal to $\mathcal{M}\left(\tau_{k}(f)\left(x_{i}\right)\right)$.

Proof. As mentioned above, $f_{*}\left(x_{i}\right) x_{i}^{-1} \in F_{k}$, since $f \in \mathcal{T}(k)$. The statement readily follows from Theorem 4.1 and Remark 4.3 with $W_{j}=f_{*}\left(x_{j}\right) x_{j}^{-1}$ and $t=2 g$.

\subsection{Vanishing condition of the Morita homomorphism}

As a lift of the Johnson homomorphism $\tau_{k}$, Morita [M2] defined a map $\tilde{\tau}_{k}: \mathcal{T}(k) \rightarrow H_{3}\left(F / F_{k}\right)$, which is called the Morita homomorphism. Furthermore, Heap Heap showed the vanishing condition of $\tilde{\tau}_{k}$ in terms of (relative) bordism theory. The purpose of this subsection is to give a simpler proof of the result (Theorem 4.6).

Let us review the map $\tilde{\tau}_{k}$. Fix $f \in \mathcal{T}(k)$. Thus, the relation $f_{*}\left(x_{j}\right) x_{j}^{-1}$ vanishes in the $k$-th nilpotent quotient of $\pi_{1}\left(T_{f}^{\gamma}\right)$. Thus, from (18), we have the canonical surjection,

$$
\phi_{f, k}^{\gamma}: \pi_{1}\left(T_{f}^{\gamma}\right) \longrightarrow \pi_{1}\left(T_{f}^{\gamma}\right) /\left(\pi_{1}\left(T_{f}^{\gamma}\right)\right)_{k} \cong F / F_{k} .
$$

Let $\left[T_{f}^{\gamma}\right] \in H_{3}\left(T_{f}^{\gamma}\right) \cong \mathbb{Z}$ be the fundamental 3-class. Then, $\tilde{\tau}_{k}(f)$ is defined to be the pushforward $\left(\phi_{f, k}^{\gamma}\right)_{*}\left(\left[T_{f}^{\gamma}\right]\right) \in H_{3}\left(F / F_{k}\right)$. It is known [M2, Heap that this $\tilde{\tau}_{k}(f)$ depends only on $f \in \mathcal{T}(k)$ and that $\tilde{\tau}_{k}$ is a lift of the Johnson map $\tau_{k}$. 
Theorem 4.6 ([Heap, Theorem 5]). Let $f \in \mathcal{T}(k)$. Then, $\tilde{\tau}_{k}(f)=0$ if and only if $f \in$ $\mathcal{T}(2 k-1)$.

Proof. First, let us make some observations. Notice from this Proposition 4.5 that $f \in \mathcal{T}(k+1)$ if and only if the pairing $\left[\left\langle x_{j_{1}}^{*}, \ldots, x_{j_{k}}^{*}\right\rangle, \iota_{*}^{\gamma}\left(\mathcal{X}_{i}\right)\right]$ in (19) is zero for any standard indexes $j_{1} \cdots j_{k}$. Furthermore, Theorem 3.2 (III) implies that $\tilde{\tau}_{k}(f)$ is zero if and only if the pairing

$$
\left\langle\mathfrak{s}_{\ell}\left(x_{s}^{*} \smile\left\langle x_{j_{1}}^{*}, \ldots, x_{j_{\ell}}^{*}\right\rangle\right), \quad\left(\phi_{f, k}^{\gamma}\right)_{*}\left(\left[T_{f}^{\gamma}\right]\right)\right\rangle
$$

is zero for any sequences $\left(s, j_{1}, \cdots, j_{\ell}\right)$ in (9), where we replace $\alpha_{j}$ by $x_{j}^{*}$.

We now complete the proof. Suppose $f \in \mathcal{T}(2 k-1)$. Then, we can define the Johnson homomorphism $\tau_{\ell}$ for $\ell \leq 2 k-2$ and have $\tau_{\ell}=0$. Hence, all the pairing $\left[\left\langle x_{j_{1}}^{*}, \ldots, x_{j_{\ell}}^{*}\right\rangle, \iota_{*}^{\gamma}\left(\mathcal{X}_{s}\right)\right]$ is zero. Note the cap product $\left[T_{f}^{\gamma}\right] \cap \alpha_{s}=\iota_{*}^{\gamma}\left(\mathcal{X}_{s}\right)$ because $H^{*}\left(T_{f}^{\gamma} ; \mathbb{Z}\right) \cong H^{*}\left(S^{1} \times \Sigma_{g, 0} ; \mathbb{Z}\right)$. Thus, the pairing in (201) is zero. Hence, $\tilde{\tau}_{k}(f)=0$. Conversely, let us assume $\tilde{\tau}_{k}(f)=0$. Then, all of the pairing (20) is zero. When $\ell=k$, Corollary 3.4 implies that the pairing (19) is zero. From Theorem 4.5, we have $\tau_{k}(f)=0$, i.e., $f \in \mathcal{T}(k+1)$; thus, we can define $\tau_{k+1}(f)$. In a similar way, we can define $\tau_{\ell}(f)$ and show $\tau_{\ell}(f)=0$ for any $\ell \leq 2 k-2$. Hence, we conclude $f \in \mathcal{T}(2 k-1)$, as desired.

Finally, we mention some results on the Morita maps $\tilde{\tau}_{k}$. Some papers [M2, Heap, Day, studied the maps. Especially, Massuyeau Mas showed an equivalence between the map $\tilde{\tau}_{k}$ and some degrees of "the total Johnson homomorphism", and give a computation of $\tilde{\tau}_{k}$ with rational coefficients, in terms of a symplectic expansion. However, our situation is with integral coefficients; if we can determine the homeomorphism type of $T_{f}^{\gamma}$, we can hope to compute $\tilde{\tau}_{k}(f)$ by using the 3 -cocycles of Theorem 3.2 (III).

\section{Expressions of some 3-cocycles}

In general, it is practically important to give explicit expressions of group cocycles. This section focuses on quotient groups of $F / F_{k}$ and gives an algorithm to describe their 3-cocycles. As mentioned in the introduction, we regard the higher Massey products as an algorithm to produce cocycles.

\section{$5.1 \quad 3$-cocycles of $F / F_{k}$}

First, let us focus on the group $F / F_{k}$ and give presentations of the 3-cocycles $\mathfrak{s}_{k}\left(\alpha_{s} \smile\right.$ $\left.\left\langle\alpha_{i_{1}}, \ldots, \alpha_{i_{k}}\right\rangle\right)$ in Theorem 3.2, when $\ell=k$ and $\ell=k+1$ with $k \geq 3$.

Using the notation in $\S 3$, given an index $i_{1} i_{2} \cdots i_{\ell}$ and $s \in \mathbb{N}$, let us define the map,

$$
\Gamma_{s i_{1} i_{2} \cdots i_{\ell}}:\left(F / F_{\ell}\right)^{3} \longrightarrow \mathbb{Z} ; \quad(x, y, z) \longmapsto c_{s}(x)\left(\sum_{j: 1 \leq j \leq \ell-1} c_{i_{1} i_{2} \cdots i_{j}}(y) c_{i_{j+1} \ldots i_{\ell}}(z)\right) .
$$

The simplest case is when $\ell=k$, in which the 3-cocycle $\mathfrak{s}_{k}\left(\alpha_{s} \smile\left\langle\alpha_{i_{1}}, \ldots, \alpha_{i_{\ell}}\right\rangle\right)$ is exactly presented as $\Gamma_{s i_{1} i_{2} \cdots i_{k}}$, since $\mathfrak{s}_{k}=$ id. Next, to get presentations of $\mathfrak{s}_{\ell}\left(\alpha_{s} \smile\left\langle\alpha_{i_{1}}, \ldots, \alpha_{i_{\ell}}\right\rangle\right)$ with $k<\ell \leq 2 k-2$, it is enough to explicitly give a function $\mathfrak{b}:\left(F / F_{\ell}\right)^{2} \rightarrow \mathbb{Z}$ such that the difference $\Gamma_{s i_{1} i_{2} \cdots i_{\ell}}-\partial_{2}^{*}(\mathfrak{b})$ is a restricted map $\left(F / F_{k}\right)^{3} \rightarrow \mathbb{Z}$.

For example, we will describe the case $\ell=k+1$. Define the map $\mathfrak{b}$ by setting

$$
\mathfrak{b}(x, y)=c_{s}(x) c_{i_{1} \cdots i_{k+1}}(y) c_{i_{k+2}}(y)+c_{s i_{1}}(x) c_{i_{2} \cdots i_{k+2}}(y)+c_{s i_{k+2}}(x) c_{i_{1} \cdots i_{k+1}}(y) .
$$

Then, as a result of the difference $\left(\Gamma_{s i_{1} i_{2} \cdots i_{k+1}}-\partial_{2}^{*} \mathfrak{b}\right)(x, y, z)$, we obtain 
Proposition 5.1. The cohomology 3-class $\alpha_{s} \smile\left\langle\alpha_{i_{1}}, \ldots, \alpha_{i_{k}}\right\rangle$ is represented by $\Gamma_{s i_{1} i_{2} \cdots i_{k}}$.

When $\ell=k+1$, the 3-class $\mathfrak{s}_{k+1}\left(\alpha_{s} \smile\left\langle\alpha_{i_{1}}, \ldots, \alpha_{i_{k+1}}\right\rangle\right)$ is represented by the map:

$$
\begin{gathered}
(x, y, z) \longmapsto \sum_{\ell=2}^{k}\left(c_{s}(x)\left(c_{i_{1} \cdots i_{\ell}}(y) c_{i_{\ell+1} \cdots i_{k+1}}(z)-c_{i_{1} \cdots i_{\ell-1}}(y) c_{i_{\ell} \cdots i_{k+1}}(z)\left(c_{i_{k+1}}(y)+c_{i_{k+1}}(z)\right)\right)\right. \\
\left.-c_{s i_{1}}(x) c_{i_{2} \cdots i_{\ell}}(y) c_{i_{\ell+1} \cdots i_{k+1}}(z)-c_{s i_{k+1}}(x) c_{i_{2} \cdots i_{\ell-1}}(y) c_{i_{\ell} \cdots i_{k}}(z)\right) .
\end{gathered}
$$

Since the length of every sequence in each term is less than $k$, this map $\Gamma_{s i_{1} i_{2} \cdots i_{k+1}}-\partial_{2}^{*} \mathfrak{b}$ can be regarded as a map from $\left(F / F_{k}\right)^{3}$.

Here, we should mention that while Igusa and Orr [IO, §10] express the 3-cocycles $\alpha_{s} \smile$ $\left\langle\alpha_{i_{1}}, \ldots, \alpha_{i_{k}}\right\rangle$ in terms of Igusa's picture, our description using Massey products is simpler and compatible with the non-homogenous complex of $G$.

Concerning the higher case $\ell>k+1$, the author attempted to describe the 3 -cocycles, but made little progress.

\subsection{Quotient groups by central elements}

This subsection deals with the situation in $\$ 4.2$ or [FS]. Namely, we fix central elements $W_{1}, \ldots, W_{t} \in F_{k} / F_{k+1}$ and let $G$ be the quotient group of $F / F_{k+1}$ subject to $W_{1}, \ldots, W_{t}$. For simplicity, let us assume $k>2$ and that there are standard sequences $I^{(j)}=\left(i_{1}^{(j)}, \ldots, i_{k}^{(j)}\right) \in \mathfrak{U}_{k}$ with $j \leq s$, which are mutually distinct, and that $W_{j}$ corresponds to the Massey product $\left\langle\alpha_{i_{1}^{(j)}}, \ldots, \alpha_{i_{k}^{(j)}}\right\rangle$ of length $k$. Such an assumption appears in discussions on higher Milnor invariant; see [Tu, $\mathrm{KN}]$.

Then, as in (5), we can easily check that the following map is well-defined and a 2-cocycle:

$$
\phi_{\Lambda_{j}}: G \times G \longrightarrow \mathbb{Z} ; \quad([X],[Y]) \longmapsto \sum_{\ell=1}^{k-1} c_{i_{1}^{(j)} i_{2}^{(j)} \ldots i_{\ell}^{(j)}}(X) c_{i_{\ell+1}^{(j)} \cdots i_{k}^{(j)}}(Y)
$$

where we represent any element of $G$ by the representative from $F / F_{k}$. Here, we should mention the 5-term exact sequence from the central extension $F / F_{k} \rightarrow G$ :

$$
0 \rightarrow H^{1}(G ; \mathbb{Z}) \stackrel{\cong}{\longrightarrow} H^{1}\left(F / F_{k} ; \mathbb{Z}\right) \longrightarrow \mathbb{Z}^{m} \stackrel{\delta^{*}}{\longrightarrow} H^{2}(G ; \mathbb{Z}) \rightarrow H^{2}\left(F / F_{k} ; \mathbb{Z}\right) .
$$

Actually, we can verify that the 2-cocycle $\phi_{\Lambda_{j}}$ corresponds to the image of $\delta^{*}$.

Now let us give some 3-cocycles of $G$.

Proposition 5.2. Let $G$ be the group $F /\left\langle F_{k}, W_{1}, \ldots, W_{s}\right\rangle$, as above. Fix $r, s \in\{1, \ldots, q\}$ such that $\left(r i_{1}^{(j)} \ldots i_{k-1}^{(j)}\right)$ and $\left(i_{2}^{(j)} \ldots i_{k}^{(j)} s\right)$ are different from other indexes $i_{1}^{\left(j^{\prime}\right)} \cdots, i_{k}^{\left(j^{\prime}\right)}$ for $j^{\prime} \leq s$. Then, the Massey product $\left\langle\alpha_{r}, \phi_{\Lambda_{j}}, \alpha_{s}\right\rangle$ is defined and is represented by the map,

$$
(x, y, z) \longmapsto c_{r}(x)\left(\sum_{\ell: 1 \leq \ell<k} c_{i_{1}^{(j)} \ldots i_{\ell}^{(j)}}(y) c_{i_{\ell+1}^{(j)} \cdots i_{k}^{(j)} s}(z)\right)-\left(\sum_{\ell: 1<\ell \leq k} c_{r i_{1}^{(j)} \ldots i_{\ell}^{(j)}}(x) c_{i_{\ell+1}^{(j)} \cdots i_{k}^{(j)}}(y)\right) c_{s}(z) .
$$

Proof. Notice the equalities,

$\alpha_{r} \smile \phi_{\Lambda_{j}}=\partial^{*}\left(\sum_{\ell: 1 \leq \ell<k} c_{r i_{1}^{(j)} \cdots i_{\ell}^{(j)}}(x) c_{i_{\ell+1}^{(j)} \cdots i_{k}^{(j)}}(y)\right), \quad \phi_{\Lambda_{j}} \smile \alpha_{s}=\partial^{*}\left(\sum_{\ell: 1<\ell \leq k} c_{i_{1}^{(j)} \cdots i_{\ell}^{(j)}}(x) c_{i_{\ell+1}^{(j)} \cdots i_{k}^{(j)} s}(y)\right)$.

Then, from the definition of the triple Massey product, we have a representative. 


\section{Acknowledgments}

The author expresses his gratitude to Teruaki Kitano and Gwénaël Massuyeau for valuable comments on this paper. He is also indebted to anonymous referees of an earlier version of this paper for providing insightful comments.

\section{A Cocycles in de Rham complexes of the iterated torus bundle}

We will describe the 2-cocycles in Theorem 3.2 as differential forms in de Rham complexes.

First, when $G=F / F_{k}$, we can verify, by induction on $k$, that the Eilenberg-MacLane space $B\left(F / F_{k}\right)$ can be realized as a $C^{\infty}$-manifold. Precisely, since $B\left(F_{k} / F_{k+1}\right)$ is the product of $N_{k}$-copies of $S^{1}$, the geometric realization of (1) implies that $B\left(F / F_{k+1}\right)$ is a universal torus bundle over $B\left(F / F_{k}\right)$.

We will address the manifold structure of $B\left(F / F_{k}\right)$ in detail. The Heisenberg (triangular) group is a good toy model from the viewpoint of the unipotent Magnus expansion $\Upsilon_{k}$. Here, we should review generalized shuffles and the image of the Magnus expansion $\mathcal{M}$. As in [CFL, §2], a sequence $\left(c_{1} c_{2} \cdots c_{k}\right) \in\{1, \ldots, q\}^{k}$ is called the resulting shuffle of two sequences $I=a_{1} a_{2} \cdots a_{|I|}$ and $J=b_{1} b_{2} \cdots b_{|J|}$ if there are $|I|$ indices $\alpha(1), \alpha(2), \ldots, \alpha(|I|)$ and $|J|$ indices $\beta(1), \beta(2), \ldots, \beta(|J|)$ such that

(i) $1 \leq \alpha(1)<\alpha(2)<\cdots<\alpha(|I|) \leq k$, and $1 \leq \beta(1)<\beta(2)<\cdots<\beta(|J|) \leq k$,

(ii) $c_{\alpha(i)}=a_{i}$ and $c_{\beta(j)}=b_{j}$ for all $i \in\{1,2, \ldots,|I|\} \quad j \in\{1,2, \ldots,|J|\}$,

(iii) each index $s \in\{1,2, \ldots, k\}$ is either an $\alpha(i)$ for some $i$ or a $\beta(j)$ for some $j$ or both.

Let the symbol $\operatorname{Sh}(I, J)$ denote the set of the resulting shuffles of $I$ and $J$. Thanks to CFL, Theorem 3.9], the image of the Magnus expansion $\mathcal{M}$ completely characterizes "the generalized shuffle relation". In fact, the image in $\mathbb{Z}\left\langle X_{1}, \ldots, X_{q}\right\rangle / \mathcal{J}_{k}$ is realized as

$$
\left\{\sum_{I=\left(i_{1} \cdots i_{n}\right)} a_{I} \cdot X_{i_{1}} \cdots X_{i_{n}} \mid \text { For any indexes } J \text { and } K, \quad a_{J} \cdot a_{K}=\sum_{L \in \operatorname{Sh}(J, K)} a_{L}\right\} .
$$

We further examine the $\mathbb{R}$-extension of the image and state Theorem A.1. Let us consider the real extension $\mathbb{R}\left\langle X_{1}, \ldots, X_{q}\right\rangle / \mathcal{J}_{k}=\mathbb{R} \otimes \mathbb{Z}\left\langle X_{1}, \ldots, X_{q}\right\rangle / \mathcal{J}_{k}$ and identify it with the Euclid space of dimension $\sum_{\ell=0}^{k-1} q^{\ell}$. Furthermore, we will define a subspace, $\operatorname{Im}\left(\mathcal{M}_{\mathbb{R}, k}\right)$, of $\mathbb{R}\left\langle X_{1}, \ldots, X_{q}\right\rangle / \mathcal{J}_{k}$ as follows. Let $\operatorname{Im}\left(\mathcal{M}_{\mathbb{R}, k}\right)$ with $k=2$ be $\mathbb{R}\left\langle X_{1}, \ldots, X_{q}\right\rangle / \mathcal{J}_{2} \cong \mathbb{R}^{q}$. Supposing the definition of $\operatorname{Im}\left(\mathcal{M}_{\mathbb{R}, k-1}\right)$, we define $\operatorname{Im}\left(\mathcal{M}_{\mathbb{R}, k}\right) \subset \mathbb{R}\left\langle X_{1}, \ldots, X_{q}\right\rangle / \mathcal{J}_{k}$ by

$$
\left\{\sum_{I=\left(i_{1} \cdots i_{n}\right)} a_{I} \cdot X_{i_{1}} \cdots X_{i_{n}} \mid \begin{array}{c}
a_{i_{1} \cdots i_{n}} \in \mathcal{M}_{\mathbb{R}, k-1}, \text { if } n<k . \\
\text { For any } J \text { and } K \text { with }|J|+|K|=n, \quad a_{J} \cdot a_{K}=\sum_{L \in \operatorname{Sh}(J, K)} a_{L}
\end{array}\right\} .
$$

Then, as in the fact [CFL, §3] that the shuffle ration is closed under the multiplication of $\left(\mathbb{Z}\left[X_{1}, \ldots, X_{q}\right\rangle / \mathcal{J}_{k}\right)^{\times}$, so is the closed set $\operatorname{Im}\left(\mathcal{M}_{\mathbb{R}, k}\right)$ under that of $\left(\mathbb{R}\left[X_{1}, \ldots, X_{q}\right\rangle / \mathcal{J}_{k}\right)^{\times}$. Hence, this $\operatorname{Im}\left(\mathcal{M}_{\mathbb{R}, k}\right)$ is a Lie group, which contains $\operatorname{Im}\left(\mathcal{M}_{k}\right)$ as a lattice. In other words, $F / F_{k}$ acts freely and properly on $\operatorname{Im}\left(\mathcal{M}_{k}\right)$.

Theorem A.1. The quotient space of $\operatorname{Im}\left(\mathcal{M}_{\mathbb{R}, k}\right)$ subject to the free action of $F / F_{k}$ is a closed connected $C^{\infty}$-manifold and is an Eilenberg-MacLane space of $F / F_{k}$. 
Proof. It is enough to show that $\operatorname{Im}\left(\mathcal{M}_{\mathbb{R}, k}\right)$ is contractible, by induction on $k$. First, if $k=2$, $\operatorname{Im}\left(\mathcal{M}_{\mathbb{R}, k}\right)$ is homeomorphic to $\mathbb{R}^{q}$ by definition. Next, consider the projection $p_{k}: \operatorname{Im}\left(\mathcal{M}_{\mathbb{R}, k}\right) \rightarrow$ $\operatorname{Im}\left(\mathcal{M}_{\mathbb{R}, k-1}\right)$. For any $x \in \operatorname{Im}\left(\mathcal{M}_{\mathbb{R}, k-1}\right), p_{k}^{-1}(x)$ can be regarded as the subspace of solutions of a linear equation, by definition of the shuffle relation. Moreover, the rank of the subspace does not depend on $x$, by (1). Thus, $p_{k}$ is a $\mathbb{R}$-vector bundle. Hence, $\operatorname{Im}\left(\mathcal{M}_{\mathbb{R}, k}\right)$ is contractible, by induction.

As a result of Theorem A.1, every $s$-form of $B F / F_{k}$ is identified with an $F / F_{k}$-invariant $s$ form of $\operatorname{Im}\left(\mathcal{M}_{\mathbb{R}, k}\right)$. Thus, via the de Rham theorem, we will describe the basis of $H^{*}\left(F / F_{k} ; \mathbb{R}\right) \cong$ $H_{\mathrm{dR}}^{*}\left(B F / F_{k}\right)$ as $F / F_{k}$-invariant $s$-forms of $\operatorname{Im}\left(\mathcal{M}_{\mathbb{R}, k}\right)$ as follows:

To state Lemmas A.2 and A.3, we need some terminology. Consider the cotangent bundle of $\mathbb{R}\left\langle X_{1}, \ldots, X_{q}\right\rangle / \mathcal{J}_{k}$, and denote by $d X_{j_{1} \ldots j_{t}}$ the dual basis corresponding to the coordinate $X_{j_{1}} X_{j_{2}} \cdots X_{j_{t}}$. Following the pullback, we regard the basis as 1 -forms in $T^{*} \operatorname{Im}\left(\mathcal{M}_{\mathbb{R}, k}\right)$. Furthermore, for $s \in\{1, \ldots, q\}$, the 1 -form $d X_{s}$ on $\operatorname{Im}\left(\mathcal{M}_{\mathbb{R}, k}\right)$ is $F / F_{k}$-invariant, and the resulting 1-cocycle in $\bigwedge^{1} B F / F_{k}$ corresponds to the $s$-th summand of the abelianization $\alpha_{s}$. Extend the $\operatorname{map} \beta_{i_{u} i_{u+1} \ldots i_{v}}$ in (5) as $\mathbb{R}\left\langle X_{1}, \ldots, X_{q}\right\rangle / \mathcal{J}_{k} \rightarrow \mathbb{R}$. In addition, for $\left(t, k_{0}\right) \in \mathbb{N}^{2}$, we prepare a set of the form,

$$
\mathcal{S}_{t, k_{0}}:=\left\{\left(k_{1}, \ldots, k_{u}\right) \in \mathbb{N}^{u} \mid u \geq 1, \quad k_{0}+k_{1}+\cdots+k_{u}=t\right\} .
$$

Lemma A.2. Fix an index $\left(j_{1}, \ldots, j_{t}\right) \in\{1, \ldots, q\}^{t}$. Define the 1-form of the formula,

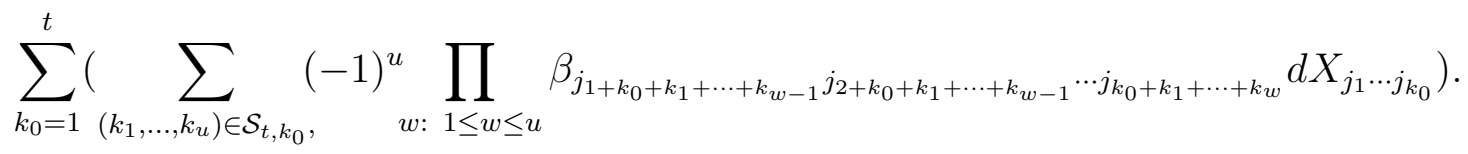

We denote this 1-form by $\gamma_{j_{1} \cdots j_{t}}$. This 1-form is $F / F_{k}$-invariant.

In what follows, we denote the sum $k_{0}+k_{1}+\cdots+k_{u}$ by $p_{u}$ for brevity.

Proof. It is enough to show that, for any $h \leq q$, the pullback $\mathcal{M}\left(x_{h}\right)^{*}\left(\gamma_{j_{1} \cdots j_{t}}\right)$ is $\gamma_{j_{1} \cdots j_{t}}$ itself. Notice, by definition, the following pullback formula:

$$
\begin{aligned}
& \mathcal{M}\left(x_{h}\right)^{*}\left(b_{j_{1} \cdots j_{k}}\right)=\beta_{j_{1} \cdots j_{k}}+\delta_{j_{k}, h} \beta_{j_{1} \cdots j_{k-1}}, \\
& \mathcal{M}\left(x_{h}\right)^{*}\left(d X_{j_{1} \cdots j_{k}}\right)=d X_{j_{1} \cdots j_{k}}+\delta_{j_{k}, h} d X_{j_{1} \cdots j_{k-1}} .
\end{aligned}
$$

Thus, the pullback $\mathcal{M}\left(x_{h}\right)^{*}\left(\prod_{w=1}^{u} \beta_{j_{1+p_{w-1}} j_{2+p_{w-1}} \cdots j_{p_{w}}} d X_{j_{1} \cdots j_{k_{0}}}\right)$ is formed as

$$
\left(\prod_{w: 1 \leq w \leq u} \beta_{j_{1+p_{w-1}} \cdots j_{p_{w}}}+\delta_{h, j_{w}} \beta_{j_{1+p_{w-1}} \cdots j_{p_{w}-1}}\right)\left(d X_{j_{1} \cdots j_{k_{0}}}+\delta_{h, j_{k_{0}}} d X_{j_{1} \cdots j_{k_{0}-1}}\right) .
$$

Denote the coefficients of $d X_{j_{1} \cdots j_{k_{0}}}$ and of $d X_{j_{1} \cdots j_{k_{0}-1}}$ by $A_{k_{0}}$ and $B_{k_{0}}$, respectively. Then, by a careful observation, we can check that

$$
(-1)^{u} \sum_{\left(k_{1}, \ldots, k_{u}\right) \in \mathcal{S}_{t, k_{0}}}\left(A_{k_{0}}-B_{k_{0}+1}\right)=(-1)^{u} \sum_{\left(k_{1}, \ldots, k_{u}\right) \in \mathcal{S}_{t, k_{0}}} \beta_{j_{1+p_{0}} \cdots j_{p_{1}}} \beta_{j_{1+p_{1}} \cdots j_{p_{2}}} \cdots \beta_{j_{1+p_{t-1}} \cdots p_{t}} .
$$

Since the sum of the left hand side running over $1 \leq k_{0} \leq t$ is equal to $\mathcal{M}\left(x_{h}\right)^{*}\left(\gamma_{j_{1} \cdots j_{t}}\right)$, we have the desired $\mathcal{M}\left(x_{h}\right)^{*}\left(\gamma_{j_{1} \cdots j_{t}}\right)=\gamma_{j_{1} \cdots j_{t}}$.

Lemma A.3. Fix an index $\left(j_{1}, \ldots, j_{k}\right)$. Then, for any $s<t \leq k$ with $(s, t) \neq(1, k)$, we have

$$
d \gamma_{j_{s} \cdots j_{t}}=\sum_{r: s \leq r \leq t-1} \gamma_{j_{s} \cdots j_{r}} \wedge \gamma_{j_{r+1} \cdots j_{t}} \in \wedge^{2} \operatorname{Im}\left(\mathcal{M}_{\mathbb{R}}\right)
$$


Proof. First, by the Leibniz rule, the left hand side $d \gamma_{j_{s} \cdots j_{t}}$ is expressed as

$$
\sum_{k_{0}=s}^{t}\left(\sum_{\left(k_{1}, \ldots, k_{u}\right) \in \mathcal{S}_{t, k_{0}}} \sum_{v=1}^{u}(-1)^{u} \beta_{j_{1+p_{0}} \cdots j_{p_{1}}} \cdots \check{\beta}_{j_{1+p_{v}} \cdots j_{p_{v+1}}} \cdots \beta_{1+j_{p_{u-1}} \cdots j_{u}} d X_{j_{1+p_{u}} \cdots j_{p_{u+1}}}\right) \wedge d X_{j_{s} \cdots j_{k_{0}}} .
$$

Here, the check $\check{\beta}_{j_{p_{v}+1} \cdots j_{p_{v+1}}}$ means the elimination of the term $\beta_{j_{p_{v}+1} \cdots j_{p_{v+1}}}$. On the other hand, the right hand side becomes

$$
\begin{aligned}
\sum_{r: s \leq r<t} & \left(\sum_{k_{0}^{\prime}: s \leq k_{0}^{\prime} \leq r}\left(\sum_{\left(k_{1}^{\prime}, \ldots, k_{u-1}^{\prime}\right) \in \mathcal{S}_{r, k_{0}^{\prime}}}(-1)^{u^{\prime}} \beta_{j_{1+s} \cdots j_{p_{1}^{\prime}}} \cdots \beta_{j_{1+p_{u-1}^{\prime}} \cdots j_{r}}\right) d X_{j_{s} \cdots j_{k_{0}^{\prime}}}\right. \\
& \left.\wedge \sum_{k_{0}^{\prime \prime}: r+1 \leq k_{0}^{\prime \prime} \leq t}\left(\sum_{\left(k_{1}^{\prime \prime}, \ldots, k_{u}^{\prime \prime}\right) \in \mathcal{S}_{t-r, k_{0}^{\prime \prime}}}(-1)^{u^{\prime \prime}} \beta_{j_{1+k_{0}^{\prime \prime}} \cdots j_{p_{1}^{\prime \prime}}} \cdots \beta_{1+j_{p_{u-1}^{\prime \prime}} \cdots j_{t}}\right) d X_{j_{r+1} \cdots j_{k_{0}^{\prime \prime}}}\right) .
\end{aligned}
$$

By replacing $r$ by $k_{0}, k_{a}^{\prime}$ by $k_{a+1}$ and $k_{a}^{\prime \prime}$ by $k_{u+a+1}$, a careful comparison deduces that this sum equal to the preceding expansion of the left hand side.

This situation is the same as the defining system, as mentioned in $\$ 3$. Note that the de Rham theorem preserves the cup product. Thus, in parallel to the main theorem 3.2 , we readily obtain the basis of the 2-cocycle of $H_{\mathrm{dR}}^{2}\left(B F / F_{k}\right)$ as follows.

Theorem A.4. The second cohomology $H_{\mathrm{dR}}^{2}\left(B F / F_{k}\right) \cong \mathbb{R}^{N_{k}}$ is spanned by the Massey products $\left\langle\alpha_{i_{1}}, \ldots, \alpha_{i_{k}}\right\rangle$ running over standard sequences $\left(i_{1} \cdots i_{k}\right) \in \mathfrak{U}_{k}$. Here, $\left\langle\alpha_{i_{1}}, \ldots, \alpha_{i_{k}}\right\rangle$ is represented by the 2-form

$$
\sum_{k_{0}=1}^{k}\left(\sum_{\left(k_{1}, \ldots, k_{u}\right) \in \mathcal{S}_{k, k_{0}}} \sum_{v=1}^{u}(-1)^{k_{0}} \beta_{j_{1+k_{0}} \cdots j_{p_{1}}} \cdots \check{\beta}_{j_{1+p_{v}} \cdots j_{p_{v+1}}} \cdots \beta_{j_{1+p_{u-1}} \cdots j_{t}}\right) d X_{j_{1+p_{u}} \cdots j_{p_{u+1}}} \wedge d X_{j_{1} \cdots j_{k_{0}}} .
$$

Proof. This resulting is immediately computed as the sum $\sum_{r=1}^{t} \gamma_{j_{1} \cdots j_{r}} \wedge \gamma_{j_{r+1} \cdots j_{t}}$ by the definition of the Massey product. Here, we should remark that the sum is $F / F_{k}$-invariant by Lemma A.2.

As a result for 3 -cocycles in $H_{\mathrm{dR}}^{3}\left(B\left(F / F_{k}\right)\right)$, Theorem 3.2 (III) and Proposition 5.1 enable us to similarly describe 3 -cocycles $\mathfrak{s}_{\ell}\left(d X_{j} \wedge\left\langle\alpha_{i_{1}}, \ldots, \alpha_{i_{\ell}}\right\rangle\right)$ as 3 -forms, where $\ell=k, k+1$. Day Day considers an extension of the Morita homomorphism from differential 3-forms of $B\left(F / F_{k}\right)$; thus, it seems interesting to observe the work from the viewpoint of Theorem A.4.

Finally, we conclude this appendix by describing some examples with $t \leq 4$.

Example A.5. (i) The 1 -form $\gamma_{a b}$ is $d X_{a b}-\beta_{a} d X_{b}$. Hence, the Massey product $\left\langle\alpha_{a}, \alpha_{b}, \alpha_{c}\right\rangle=$ $\gamma_{a b} \wedge \gamma_{c}+\gamma_{a} \wedge \gamma_{b c}$ is expressed as $d X_{a} \wedge d X_{b c}+d X_{a b} \wedge d X_{c}-\beta_{a} d X_{b} d X_{c}-\beta_{b} d X_{a} d X_{c}$.

(ii) Next, when $t=3$, the 1 -form $\gamma_{a b c}$ is $d X_{a b c}-\beta_{c} d X_{a b}-\beta_{b} \beta_{c} d X_{a}+\beta_{b c} d X_{a}$. Hence, the Massey product $\left\langle\alpha_{a}, \alpha_{b}, \alpha_{c}, \alpha_{d}\right\rangle$ is formulated as

$$
\begin{gathered}
\left(d X_{a b c}-\beta_{c} d X_{a b}-\beta_{b} \beta_{c} d X_{a}+\beta_{b c} d X_{a}\right) \wedge d X_{d}+\left(d X_{a b}-\beta_{a} d X_{b}\right) \wedge\left(d X_{c d}-\beta_{c} d X_{d}\right) \\
+\beta_{a} \wedge\left(d X_{b c d}-\beta_{d} d X_{b c}-\beta_{c} \beta_{d} d X_{b}+\beta_{c d} d X_{b}\right) .
\end{gathered}
$$

(iii) Next, when $t=4$ and $\left(j_{1}, j_{2}, j_{3}, j_{4}\right)=(a, b, c, d)$, the 1 -form $\gamma_{a b c d}$ is

$$
d X_{a b c d}-\beta_{d} d X_{a b c}+\beta_{c} \beta_{d} d X_{a b}+\beta_{c d} d X_{a b}-\beta_{b c d} d X_{a}-\beta_{b c} \beta_{d} d X_{a}-\beta_{b} \beta_{c d} d X_{a}-\beta_{b} \beta_{c} \beta_{d} d X_{a}
$$

Then, $\left\langle\alpha_{a}, \alpha_{b}, \alpha_{c}, \alpha_{d}, \alpha_{e}\right\rangle$ can be similarly computed as $\gamma_{a b c d} \wedge \gamma_{e}+\gamma_{a b c} \wedge \gamma_{d e}+\gamma_{a b} \wedge \gamma_{c d e}+$ $\gamma_{a} \wedge \gamma_{b c d e}$ 


\section{References}

[BC] P Benito, D de-la-Concepción, An overview of free nilpotent Lie algebras Comment. Math. Univ. Carolin, ,3 (2014) 325-339

[Bro] K. S. Brown, Cohomology of Groups, Graduate Texts in Mathematics, 87, Springer-Verlag, New York, 1994.

[CFL] K. T. Chen, R. H. Fox, R. C. Lyndon, Free differential calculus IV, the quotient groups of the lower central series, Ann. of Math. 68 (1958), 81-95.

[CGO] T. Cochran, A. Gerges and K. Orr, Dehn surgery equivalence relations on three-manifolds, Math. Proc. Cambridge Philos. Soc. 131 (2001) 97-127.

[Day] M. B. Day, Extending Johnson's and Morita's homomorphisms to the mapping class group, Algebr. Geom. Topol., 7:1297-1326, (2007).

[FS] R. Fenn, D. Sjerve, Massey products and lower central series of free groups, Canad. J. Math. 39 (1987) $322-337$.

[GL] S Garoufalidis, J Levine, Tree-level invariants of three-manifolds, Massey products and the Johnson homomorphism, from: "Graphs and patterns in mathematics and theoretical physics, Proc. Sympos. Pure Math. 73, Amer. Math. Soc., Providence, RI (2005) 173-203

[GG] C. K. Gupta, N. D. Gupta, Generalized Magnus embeddings and some applications, Math. Z. 160 (1978), $75-87$.

[Hall] M. Hall, A basis for free Lie rings and higher commutators in free groups, Proceedings of the American Mathematical Society, 1 (5): 575-581,

[Heap] A. Heap. Bordism invariants of the mapping class group, Topolog, 45(5): 851-886, 2006.

[IO] K. Igusa, K. Orr, Links, pictures and the homology of nilpotent groups, Topology 40 (2001), 1125-1166.

[Ki] T. Kitano, Johnson's homomorphisms of subgroups of the mapping class group, the Magnus expansion and Massey higher products of mapping tori, Topology Appl. 69 (1996), no. 2, 165-172.

[Joh1] D. Johnson, An abelian quotient of the mapping class group $\mathcal{I}_{g}$, Math. Ann. 249 (1980) 225242.

[Joh2] D. Johnson, A survey of the Torelli group, Contemporary Mathematics 20 (American Mathematical Society, Providence, RI, 1983) 165-179.

[Kra] D. Kraines, Massey higher products, Trans. Amer. Math. Soc. 124 (1966) 431-449.

[KN] H. Kodani, T. Nosaka, Milnor invariants via unipotent Magnus embeddings, preprint.

[Mil] J. Milnor, Isotopy of links, in "Algebraic geometry and topology. A symposium in honor of S. Lefschetz", 280-306, Princeton University Press, Princeton, NJ, 1957

[MKS] W Magnus, A Karrass, D Solitar, Combinatorial Group Theory, Interscience Publ., New York (1966)

[Mas] G. Massuyeau, Infinitesimal Morita homomorphisms and the tree-level of the LMO invariant, Bull. Soc. Math. France 140:1 (2012) 101-161.

[M1] S. Morita, The extension of Johnsons homomorphism from the Torelli group to the mapping class group, Invent. Math. 111 (1993), 197-224.

[M2] S. Morita, Abelian quotients of subgroups of the mapping class group of surfaces, Duke Math. Journal 70 (1993) 699-726.

[O1] K. Orr, Homotopy invariants of links, Inventiones Math. 95 (1989), 379-394.

[O2] K. Orr, Link concordance invariants and Massey products, Topology in 1991

[Po] R. Porter, Milnor's $\bar{\mu}$-invariants and Massey products, Trans. Amer. Math. Soc. 275 (1980), 39-71.

[Tu] V.G.Turaev, Milnor's invariants and Massey products, J. SovietMath. 12 (1979), 128-137.

[Witt] E. Witt; Treue Darstellung Liescher Ringe, Journal für die Reine und Angewandte Mathematik, 177 (1937), 152-160.

DEPARTMENT OF MATHEMATICS TOKYO INSTITUTE OF TECHNOLOGY 2-12-1 OOKAYAMA , MEGURO-KU TOKYO 152-8551 JAPAN 